\title{
TAREAS DE PLANIFICACIÓN DEL MÓDULO «LA ENERGÍA Y LOS RECURSOS ENERGÉTICOS» EN EL MARCO DE LA FORMACIÓN DEL PROFESORADO
}

\author{
HERNÁNDEZ ABENZA, L.M. \\ Departamento de Didáctica de las Ciencias Experimentales. EU de Magisterio. \\ Universidad de Murcia.
}

\begin{abstract}
SUMMARY
In this paper, an alternative methodological framework to the traditional teaching of Energy is proposed. In framework we intend to give an answer to the difficultes involved in this problem area with respect to the learning of this energetic model. The elements which support this curriculum proposal are described and the characteristics which are more relevant are mentioned.
\end{abstract}

\section{INTRODUCCIÓN}

El planteamiento tradicional de la enseñanza de la «energía» en los niveles de educación secundaria siempre ha venido delimitado por un marco científico-físico consistente en la exposicion de enunciados y actividades sobre definiciones y principios, muy alejado del marco social y tecnológico en el que el alumno se relaciona con la energía a través de su entomo cotidiano, lo que ha provocado una problemática didáctica de la enseñanza de ésta, cuyo análisis y solución se hace conveniente desde el ámbito de la formación inicial del profesor. En este sentido, hemos emprendido la tarea de planificación y desarrollo del módulo «La Energía y los Recursos Energeticos» con alumnos de tercer año de la especialidad de Ciencias de la EU de Magisterio de la Universidad de Murcia, en el marco de un proyecto de investigación educativa (Pro 1989) que tiene como eje central la integración de los contenidos científicos (disciplinas científicas), los contenidos didácticos (didácticas especiales) y los contenidos profesionales («Prácticas de Enseñanza») y que trata de dar respuesta a la desconexión teoría-práctica, problema clásico en la fornación del profesorado de Ciencias.

La estructuración del presente trabajo comprende un primer apartado ("Tareas de preparación del módulo»), que recoge los elementos de referencia que hemos considerado conveniente analizar para fundamentar el proceso de planificación y diseño del módulo. A continuación se describen las tareas de planificación lievadas a cabo, una de las cuales (diseño de estrategias didácticas) viene concretada en el último apartado.

\section{TAREAS DE PREPARACIÓN DEL MÓDULO}

\section{Elementos de referencia}

Seleccionamos cuatro elementos de referencia que fueron objeto de un análisis en profundidad, deI cual resumimos aquí las conclusiones más significativas para así poder establecer implicaciones concretas para nuestras tareas de planificación.

\section{Consulta a profesores de educación secundaria}

El trabajo interactivo con un grupo de 50 profesores de enseñanza secundaria, a través de cuestionarios (como el del cuadro X) y entrevistas sucesivas, contribuyó, de manera significativa, a clarificar nuestra tarea de análisis de los contenidos, en función de cómo ellos seleccionaban y secuenciaban los correspondientes al currículo de secundaria. En general, el tema de «Recursos Energéticos" lo consideran importante por su relación con la vida cotidiana del niño, aunque, «por falta de tiempo», no lo contemplan o es tratado de forma anecdótica. En cuanto a planteamientos didácticos, están concienciados de la existencia de concepciones previas en los alumnos, pero no es un material integrado en sus procesos de $\mathrm{E} / \mathrm{A}$.

\section{Análisis de las propuestas oficiales}

El trabajo de revisión, centrado principalmente en el DCB del Proyecto de Reforma, consistió básicamente en analizar el tratamiento científico y didáctico dado al conjunto de estos contenidos. Destacamos aquí, por un lado, el marco psicopedagógico (en la línea constructi- 
Cuadro I

CONSULTA A PROFESORES

\begin{tabular}{llllll}
\hline BLOQUE TEMÁTICO: & ¿En qué nivel & Grado de & & Dificuitad & Dificultad \\
ENERGIA & debería estu- & importancia & de tratamien- & de aprendi- & que usarias \\
diarse? & en ese nivel & to didáctico & zaje & (máximo tres)
\end{tabular}

Unidades temáticas:

Concepto de «energía»

Ejemplo de otras unidades temáticas incluidas en el cuadro son las siguientes: unidades, clases, fuentes de «energían, centrales eléctricas indicadas por separado), aspectos socioeconómicos (conceptos básicos relacionados con la producción y consumo de «energían) aspectos socioambientales,...

vista) en el que parece buscar su fundamentación y, por otro, el potenciar la tendencia del alumno a explorar la realidad del mundo físico de su entorno, ya que que han de estar preparados para comprender una sociedad fuertemente impregnada de elementos científicos y tecnológicos; los objetivos 2,7 y 9 del área y los BT 3 y 14 van en ese sentido desde la óptica de la «energía».

\section{Actualización científico-didáctica}

Ha sido realizada una extensa revisión bibliográfica, que incluye los ámbitos científico y educativo, a través de la cual se han evidenciado problemas como:

1) Problemática en la contextualización de la «energía» debido al proceso de abstracción que el concepto lleva implícito, sobre el que se atribuyen desde significados «funcionales» (Watts 1983) o significados físicos con las definiciones clásicas (Warren 1982), hasta el significado material por el que la «energía» es un concepto abstracto que no admite definición operativa (SexI 1981, Feynman 1971).

2) Existe una línea importante de investigación referida a las concepciones o ideas previas de los alumnos de los diversos niveles educativos en la que aparece reflejada la problemática anterior, tanto en las causas y caracterización de dichas concepciones como en las discrepancias de las diversas propuestas didácticas (algunas de ellas recogidas por Hierrezuelo 1986 y Sevilla 1986) para la reestructuración y evolución de las mismas. Un análisis global de los resultados encontrados nos permiten afirmar que vienen mucho más influidas por el marco de su vida cotidiana (Bachélerie 1982, Watts 1983, García Gómez et al. 1989) que por el marco científico de los contenidos que van recibiendo sobre la "energía», aunque a medida que pasamos a niveles superiores de enseñanza se observa que sus ideas previas van apareciendo a través de un lenguaje académico, a veces adquirido por imitación, pero que todavía lleva implícito un «sustrato» significativo de errores conceptuales, relacionados sobre todo con el concepto de «energía» (Watts y Gilbert 1983, Duit 1983) y con la aplicación del principio de conservación (Driver y Warrington 1985, Solomon 1985 , Elise 1988, Koballa 1989). Por nuestra parte, la exploración realizada a una muestra de alumnos de diversos niveles educativos confirma estos resultados.

\section{Consulta a especialistas científicos}

El cuestionario del cuadro II fue pasado a una pequeña muestra de 10 científicos relacionados con el campo de la «energia». Su aportación principal fue la obtención por nuestra parte de una lista de contenidos básicos para el tema de «Recursos Energéticos» y, por otro lado, su opinión sobre la posibilidad de utilización de los medios de comunicación como un material diđáctico alternativo a los habituales.

\section{Cuadro II}

\section{CUESTIONARIO PARA CONSULTA A ESPECIALISTAS}

A. ¿Qué opinión le merece et nivel científico-cultural de las informaciones (noticias, reportajes, comunicados de empresas energeticas...) que aparecen en los medios de comunicación social sobre la problemática general de la producción y uso de la "energía" asf como de otros aspectos (socioeconomicos, impacto ambiental...) relacionados con ella?

¿Cree que dicho nivel de información es adecuado al nivel cultural que posee el «ciudadano medio»?

En caso de no adecuación, ¿cómo considera que se podría subsanar esta «barrera» entre los dos niveles?

B. ¿Qué conocimientos básicos, para cada una de las fuentes de «energía» expresadas a continuación, considera que debería poseer el «ciudadano medio» para poder estar al dia de los avances en este campo y para poder comprender las informaciones, al respecto, de los medios de comunicación social: a) energía a partir del petróleo, carbón y gas natural; b) energia nuclear de fision; c) energía hidroeléctrica; d) energía solar. ¿Que otros tipos de fuente de "energía» considera potencialmente significativos para que en un futuro puedan ser verdaderas alternativas a las actuales fuentes de "energía»?

C. A su criterio, ¿cuál cree que poơría ser el «mapa» energético en el año 2000 en nuestro país y en el ámbito mundial? (mapa energético en cuanto a la utilización de fuentes de «energias).

\section{IMPLICACIONES PARA LAS TAREAS DE PLANIFICACIÓN}

Todos los elementos de referencia analizados demandan la necesidad de una educación energética relacionada con el entorno social del alumno, como característica principal, y basada en los siguientes puntos:

1. Conveniencia de una propuesta didáctica sobre la «energía» que parta, en su nivel inicial, del estudio de los 
recursos energéticos utilizados por el hombre, pues es el marco más relacionado con el entorno cotidiano del alumno (según profesores consultados y bibliografía revisada), de tal forma que es importante abordar las transformaciones de la «energía» desde una perspectiva real proveniente de las instalaciones energéticas existentes (especialistas científicos).

2. Como consecuencia de la implicación anterior y teniendo en cuenta el DCB y la bibliografía consultada, surge la necesidad de una formación sobre los aspectos sociales (económicos y ambientales) que derivan de la utilización de los recursos energéticos.

3. A partir de este primer nivel de tratamiento del tema, se pueden abordar, en un segundo nivel, los conceptos y principios energéticos básicos que están sustentando, desde el punto de vista científico, la utilización de esos recursos por el hombre. Esta secuenciación viene apoya* đa por el análisis bibliográfico y la consulta a científicos.

4. Este planteamiento propuesto para la enseñanza de la "energía» debe suponer un cambio metodológico del profesor cuya diferenciación clave es la libertad de toma de decisiones respecto a Ia planificación y desarrollo del tema, en función de los condicionantes de tipo científico (naturaleza del tema), de tipo didáctico (caracterización del alumnado y recursos disponibles) y de tipo social (caracterización del entorno al que pertenecen los alumnos) que encuentre en su aula concreta de trabajo.

\section{TAREAS DE PLANIFICACIÓN DEL MÓDULO}

En función de las implicaciones anteriores podemos descodificar los aspectos más importantes de nuestras tareas de planificación. En el cuadro III aparece un esquema de la secuenciación general de los contenidos.

\section{Cuadro III}

\section{ESQUEMA GENERAL DE SECUENCIACIÓN DE LOS NIVELES DEL PROGRAMA DEL MÓDULO}

Nivel l. Fuentes de "energia" y su utilización en la vida cotidiana

- ¿De donde se obtiene la «energía» que consumimos? Estudio de los materiales de partida (combustibles fósiles, material nuclear, agua, sol, residuos...).

- ¿Cómo se obtiene dicha «energfan?: tratamiento del petróleo, estudio de centrales eléctricas...

$-\succsim$ Qué incidencias tienen los procesos de obtención de «energía» en nuestra vida cotidiana? Aspectos sociales (economicos, ambientales y políticos).

- ¿En qué utilizamos la «energía» obtenida? Consumo de «energía».

Nivel 2. Bases cientificas que sustentan la utilización de fuentes de "energía".

- ¿Qué hemos aprendido de la «energía» en el nivel 1 ? Clases de "energía» y su categorización.

- ¿Qué principios gobiernan los cambios de «energía»? Transformaciones de la «energía», procesos asociados, principios de conservación y degradación.
- ¿Qué es la «energían? Diferenciación de conceptos relacionados, marco conceptual de la «energia».

Nivel 3. La "energía" y los recursos energéticos en la educación secundaria

- ¿Qué referencias hay que considerar para la planificación? Elementos de referencia (actualización científica y didáctica, análisis de propuestas...) para la fundamentación de la planificación.

- ¿Cómo planificar el proceso de E/A? Tareas de planificación.

- ¿Cómo contrastar la validez de nuestra planificación? Aplicación en el aula de enseñanza secundaria de módulos de aprendizaje diseñados por nuestros alumnos. Evaluación.

\section{ANÁLISIS DE LOS CONTENIDOS DEL MÓDULO}

\section{Contenidos físicos}

El proceso de análisis seguido, que consistió en la selección de los contenidos, grado de relevancia y su diferenciación conceptual, se concreta en los Mapas de conceptos I y 2 . Consideramos como aspecto relevante del módulo la comprensión y aplicación del principio de conservación, el cual ha de ser trabajado paralelamente con el principio de degradación de la «energía». En otro nivel importante de relevancia, se encontraría el tema de las fuentes de «energía» y su utilización, pues en este campo es donde nuestros alumnos poseen la mayoría de

Mapa de conceptos I

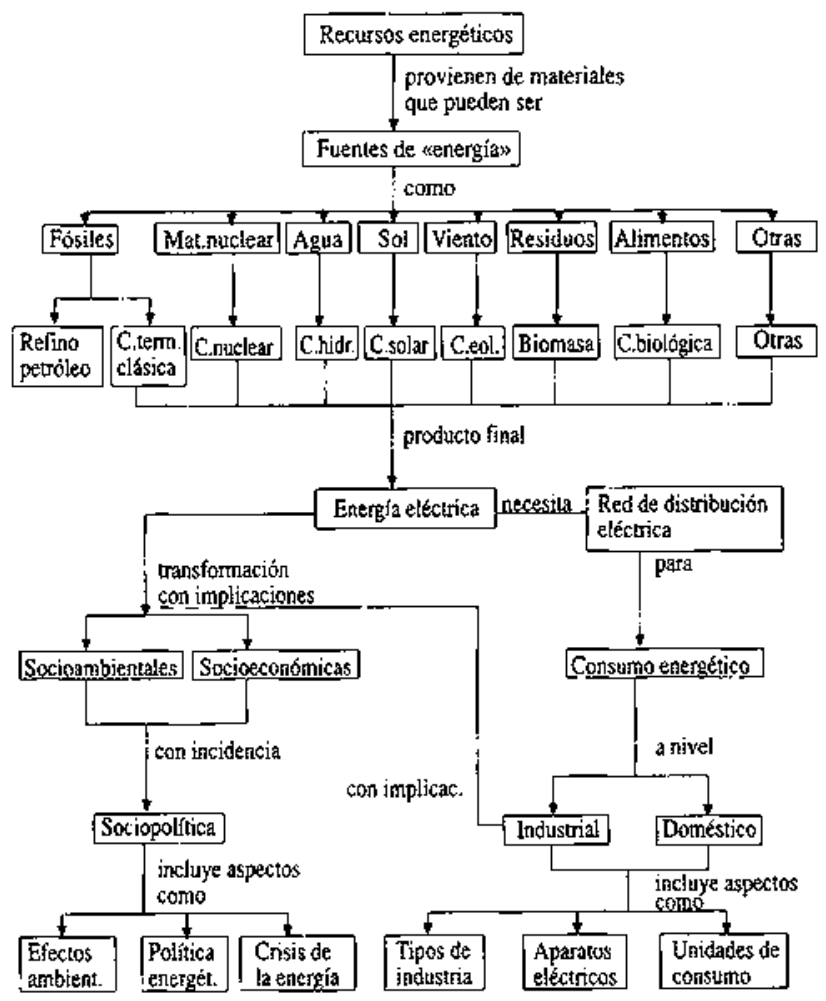


Mapa de conceptos II

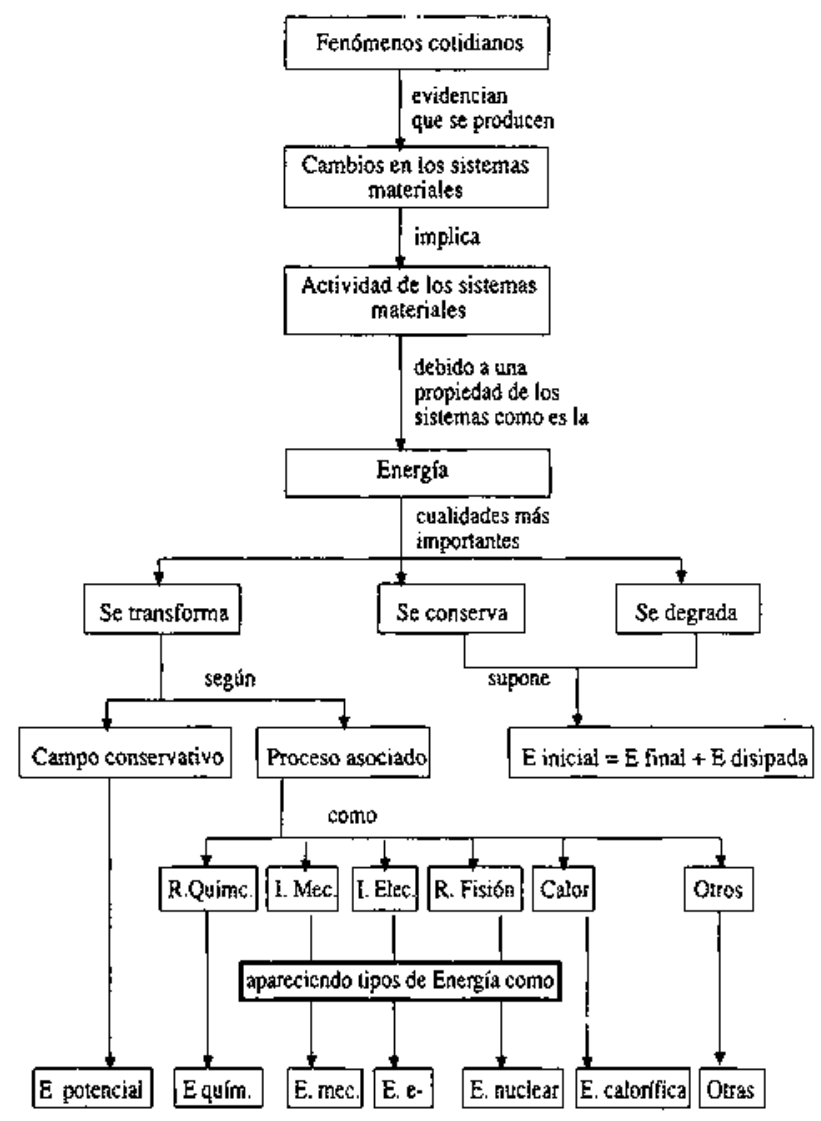

sus ideas previas y es desde donde podemos partir para iniciar cualquier propuesta curricular sobre la «energía». Los aspectos económicos y ambientales completan la información que los alumnos deben poseer para comprender el mundo científico y tecnológico que les rodea. En el mismo nivel estaría el apartado de consumo de «energía».

\section{Contenidos didácticos}

Considerando que nuestro proyecto de trabajo se fundamenta en la necesidad de una integración científica y didáctica en la formación inicial del profesorado, hemos tomado como eje central los contenidos científicos de la física para establecer una serie de módulos de aprendizaje donde aparecen distribuidos, en función de la naruraleza de cada módulo, el conjunto de contenidos didácticos que, desde la física, creemos que deben adquirir los futuros profesores de Ciencias. En el caso de nuestro módulo, la relación de contenidos didácticos, que viene inferida a través del Mapa de conceptos 3, comprende, a nivel general, elementos de referencia para fundamentar las tareas de planificación, el análisis de determinados materiales y recursos didácticos (como MAV y medios de comunicación escritos) y, finalmente, el estudio de tareas de planificación.
Mapa de conceptos III

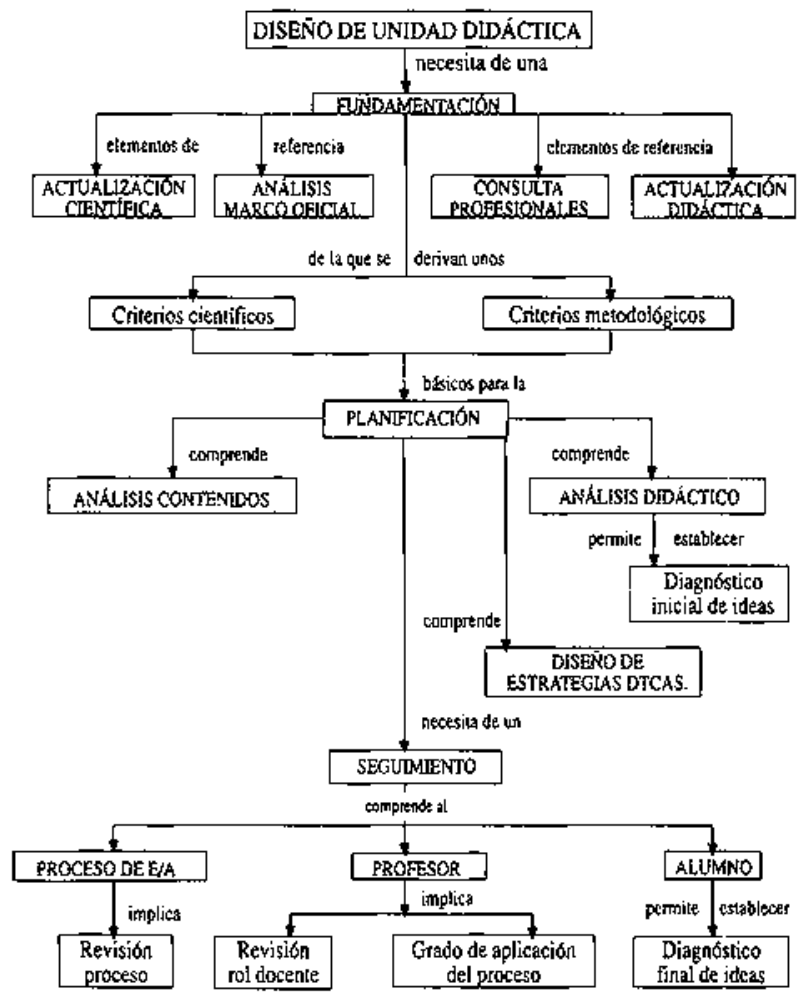

\section{Contenidos de formación práctica}

El programa de Prácticas de Enseñanza para el tercer curso, desarrollado durante los dos meses últimos del segundo trimestre, incluye un plan de acción en el aula de Ciencias con un grado creciente de intervención autónoma del alumno en prácticas, para así lograr una interacción con los niños lo más ađecuada posible. Comprende la observación đe elementos y características del aula, una segunda fase de realización de actividades puntuales (por iniciativa del alumno-profesor y a propuesta de los tutores), y una tercera fase que consiste en el diseño y puesta en práctica de una unidad didáctica. Después del período de prácticas, para completar ideas del tercer nivel del módulo, se contrastan y evalúan las UD aplicadas en el aula por los alumnos.

\section{ANÁlISIS DIDÁCTICO: CARACTERIZACIÓN DE LOS ALUMNOS DE CARA AL MÓDULO «LAENERGÍA Y LOS RECURSOSENERGETICOS»}

Los resultados encontrados en la aplicación sucesiva, durante dos cursos académicos, de instrumentos de exploración inicial como entrevistas y cuestionarios (como, por ejemplo, en el cuadro IV) nos sugieren un perfil aproximado del futuro profesor, respecto a este módulo de aprendizaje, concretado en las siguientes características: 
Cuadro IV

\section{INSTRUMENTOS PARALAEXPLORACIÓNINICIAL DE IDEAS}

(Incluimos solamente algunas cuestiones de cada apartado del cuestionario realizado)

\section{Conocimientos científicos}

1. Comenta brevemente qué es la «energía» para ti. Pon un ejemplo, mediante un dibujo, que represente tu idea al respecto.

2. Cita todas las fuentes de cenergía» que sepas.

3. ¿Qué crees que puede contaminar más el ambiente: una central de «energía» solat o una central térmica de carbón? ¿Por qué?

\section{Actitudes $\mathrm{e}$ intereses}

1. Puntúa de 0 a 3 cada una de las siguientes opiniones sobre la «energía» según estés más o menos de acuerdo con ellas. La «energía» es: necesaria, contaminante, importante para el progreso, se podria vivir sin ella, peligrosa, favorece la salud de las personas.

2. Cita cinco noticias referentes a la «energía» o a los recursos energéticos que recuerdes haber leido, oído o visto en prensa, radio o televisión.

\section{Pruebas de transferencia conceptual}

a) Cuestiones de la vida cotidiana:

1. Describe un ejemplo de la vida cotidiana en donde se cumpla el principio de conservación de la «energía» y justifica por qué crees qque se cumple.

2. Después de haber visto el siguiente vídeo documental (sobre una central hidroeléctrica, vídeo sin sonido), responde a estas cuestiontes: ¿Qué es la instalación que aparece en el río? ¿Para qué sirve? ¿Qué partes principales contiene la instalación? Indica si has visto personalmente alguna instalación parecida y di si observaste alguna diferencia con la mostrada en el vídeo

b) Completa el siguiente cuadro y justifica las respuestas negativas:

Objeto y situación de medida

¿El objeto posee E?

¿Qué clase o clases de $\mathrm{E}$ ?

Si se pudiera utilizar como fuente de $\mathrm{E}$, ¿qué clases de $\mathrm{E}$ aparecerfan?

\begin{abstract}
Bola en nuestra mano
con brazo levantado

El resto de los objetos que completan el cuadro son los siguientes: leche en un vaso a temperatura ambiental, bola en el bolsillo del pantalon, bola dejada caer (a mitad de recorrio y al llegar al suelo), bola impulsada a) suelo (a mitad de recorrido y al llegar al suelo), piedra inmóvil en ef stelo, agua tirviendo, un trozo de manzana, el Sol, la Luna, un trozo de carbón ardiendo, un trozo de hieno, un trozo de uranio.
\end{abstract}

\section{Contenidos didácticos}

1. De la relación de contenidos sobre là «energía» (fotocopia), elegir uno de ellos y planificar y diseñar una unjdad didáctica (incluyendo todos los elementos didácticos que consideres conveniente) para desarrollarla en una aula de? nivel que elijas del ciclo 12-16.

2. Anślisis de noticias de prensa relacionadas con la cenergía” y los recursos energéticos. Se reparten diversas noticias y se pide a los alumnos: a) Lista de conceptos científicos y técricos. Señalar los nocomprensibles. b) Resumir en 4 o 5 líneas la idea clave de la noticia. c) Relacionar de la mejor forma organizada posible (desde el punto de vista científico) los diversos conceptos expuestos en la lista (mediante mapade conceptos, esquema, etc.).d) Revisión bibliográfica sobre aspectos didácticos y científicos relacionados con el contenido de la noticja (revision en grupos de trabajo). Se debate en gnupo de trabajo sobre todos los aspectos analizados en el contenido de la noticia y posterior puesta en común.
1. Deficiencias significativas en su formación científica y dificultades en la transferencia de aprendizaje con un nivel mínimo de aplicación đe las ideas adquiridas. Así, para explicitar conceptos básicos en este módulo, los alumnos adoptan en general formulaciones de tipo «libresco", bien definiciones clásicas o apoyándose en ecuaciones matemáticas. Es interesante comprobar que cuando se expresan sin ayuda de estas formulaciones es cuando dejan entrever las confusiones que realmente tienen respecto a estos conceptos. En cuanto a aspectos relacionados con los recursos energéticos, la influencia manifiesta de su entorno cotidiano y la de los medios de comunicación no elimina la problemática conceptual al respecto.

2. Con referencia a las actitudes e intereses frente al tema de la «energía» destaca el balance positivo en sus respuestas, siendo menos valorado, aunque son citados en un porcentaje significativo, los adjetivos de tipo negativo ("contaminante», «peligrosa»...).

3. Los resultados del conjunto de instrumentos utilizados para la exploración de iđeas en el ámbito didáctico reflejan globalmente una formación didáctica de nuestros alumnos con distorsiones importantes, fruto de la interacción de ideas de su formación tradicional y la recibida en las materias didácticas de la especialidad de Ciencias. Así, por ejempio, la mayoría de la muestra contempla la exploración de ideas previas como primer elemento didáctico de su diseño metodológico pero sin justificar su función y su relación con el resto de elementos didácticos, los cuales suelen ser los propios de una formación didáctica clásica (objetivos, desarrollo de contenidos y actividades), siendo la «explicación de ideas» el recurso más utilizado y el que dirige el enfoque de sus planteamientos metodológicos.

4. Respecto al marco profesional, las ideas de los alumnos-profesores sobre aspectos didácticos (expresadas anteriormente) y sas experiencias previas en prácticas de aula en primero y segundo curso reflejan un papel clásico de profesor protagonista fundamental de la acción escolar, papel que está lejos del perfil de profesor interactivo y dinamizador del aprendizaje de sus alumnos.

\section{Diseño de estrategias didácticas}

El que uno de nuestros principios metodológicos básicos de nuestro proyecto sea el de integración de contenidos en la formación de nuestros alumnos hace recomendable, en nuestro proceso de E/A, la aplicación de secuencias parecidas a las que los futuros profesores han de llevar a cabo con sus alumnos de secundaria. En este sentido hemos de destacar la importancia de que el profesor conozca las ideas previas de los alumnos sobre la «energía» y respete el proceso de construcción de las mismas por parte de éstos, modelo de E/A (Osborme y Witthock 1985) básicamente fundamentado en la teoría del aprendizaje constructivista. La visión de paralelismo de nuestra propuesta con los planteamientos del proyecto CLISP (AAVV 1987, Driver 1988) aparecería deformada sin mencionar el proceso, lógicamente llevado a 
cabo, de flexibilidad y adecuación al entorno educativo concreto en el que estamos trabajando.

El proceso de integración científico-didáctico aparece, en el caso de nuestro módulo, de una forma secuenciada a lo largo del desarrollo del programa propuesto y bajo dos formas de trabajo: de manera indirecta, en el desarrollo de los contenidos físicos (niveles 1 y 2) y, de forma directa, en la propuesta que se hace en el tercer nivel del programa. Es evidente que el planteamiento metodológico implícito en el desarrollo de los contenidos físicos, Ia forma de trabajar las distintas fases del proceso de $E$ / A, el papel adoptado por el profesor, los recursos y materiales utilizados... son elementos del ámbito didáctico en relación con los cuales el profesor debe tratar de concienciar a sus alumnos de la importancia de su conocimiento y adquisición. En función del resultado de este proceso indirecto de adquísición de contenidos didácticos, se desarrolla el tercer nivel del programa con el fin de organizar, clarificar y completar las ideas de los alumnos respecto al marco didáctico de la «energía».

\section{Diseño de estrategias de evaluación}

Respecto al seguimiento del módulo, el proceso de E/A diseñado lleva implícito la propuesta de estrategias de evaluación para diagnosticar la evolución de las ideas de los alumnos y la eficacia de sus trabajos, así como el grado de coherencia, respecto al modelo didáctico de referencia, de la aplicación del diseño del módulo por parte del profesor. Esta recogida de información es completada mediante el empleo de determinados instrumentos como protocolos de observación, entrevistas, diarios y grabación en circuito cerrado entre otros. Describimos brevemente algunos de ellos:

\section{Protocolos de observación}

Se diseñaron protocolos de observación para las diversas fases del proceso, confeccionados en función de la fase observada (ver ejemplo en cuadro V). Los datos se obtienen directamente del desarrollo de la sesión y a través de circuito cerrado de televisión, el cual permite también la contrastación de los datos recogidos con el conjunto de instrumentos utilizados, aportando información sobre la fiabilidad de los mismos.

\section{Diario del profesor}

Recoge tres apartados básicos para cada sesión (intenciones del profesor, desarrollo real de la sesión y reflexión sobre lo acontecido). El diario es realizado por el propio profesor al final de la sesión y su información es contrastada con la del diario de los alumnos.

\section{PROTOCOLO DE OBSERVACIÓN PARA FASE DE ORIENTACIÓN}

\begin{tabular}{|c|c|c|}
\hline \multicolumn{2}{|l|}{ Dfa: } & PROFESOR \\
\hline Profesor: & & DESARROLLO \\
\hline Modulo: & 12345 & 123456 \\
\hline \multicolumn{3}{|l|}{ Curso: } \\
\hline \multicolumn{3}{|c|}{ Asignatura: } \\
\hline Fase: ORI & & \\
\hline
\end{tabular}

\section{INTERACCIÓN}

1234

\section{ALUMNOS INTERACCIÓN}

1234

\section{ACTIVIDAD}

123

\section{DESCRIPCIÓN DE ACTIVIDADES}

\section{Explicación verbal}

Planteamiento de cuestiones

MAV

Otras

Lista de tópicos contenidos en el cuadro:

DEL PROFESOR

Finałidad: a) iniciación al tema, b) motivación, c) orientación, d) explicitación, e) problemática específica del tema. Desarrollo: a) contempla aspectos generales de conteniơos, b) idem de la organización, c) contempla relación con otras partes de la asignatura, d) explicita con claridad las fases de secuenciacion del proceso de E/A, e) conecta el tema con hechos de la vida cotidiana, f) otros. Interacción: $a$ ) pide a los alumnos sugerencias sobre aspectos del móduło, b) recoge preguntas espontáneas de los alumnos, c) informa sobre bibliograffa relacionada con el módulo, d) otros.

DE LOS ALUMNOS

Interacción: a) siguen con atencion el desarrollo de la actividad, b) piden clarificación sobre lo explicado, c) demandan más información, d) sugieren aiternativas y las fundamentan.

DE LA ACTIVIDAD

a) Adecuada para iniciar el tema, b) adecuada para motivar a los alumnos, $c$ ) adecuada para orientarlos. 


\section{Entrevistas a alumnos}

Permiten recabar información específica đel trabajo de cada grupo y de sus dudas y planteamientos respecto a los contenidos de la fase que se esté trabajando en ese momento del curso. Se realizan en momentos claves del proceso de $\mathrm{E} / \mathrm{A}$ previamente determinados por el profesor o en función del desarrollo del módulo.

\section{TAREAS DE DISEÑO DEL MÓDULO}

En cada uno de los tres niveles del programa se desarroIlaron las fases (explicitación, reestructuración, aplicación y revisión) del proceso de E/A diseñado, salvo la fase de orientación que fue común y planteada en el inicio del curso. Debido a la extensión del trabajo realizado, resumimos aquí algunas de las actividades que mejor reflejan el aspecto de integración de los contenidos sobre formación del profesorado.

\section{Fase de orientación}

- Materiales didácticos y organización: Noticias de prensa sobre recursos energéticos, propuestas ministeriales y libros de texto sobre el tema. Se organizan debates en grupos de trabajo sobre los materiales seleccionados y se hace una posterior puesta en común con intervención final del profesor para justificar el contenido y enfoque del módulo.

- Justificación: Con un material, cuidadosamente seleccionado, se pretende la motivación y orientación de nuestros alumnos respecto al módulo, despertando su interés sobre los problemas reales en torno a la «energía» y ofreciéndole una visión alternativa, más cercana a su vida cotidiana, que la que brindan los actuales materiales didácticos y documentos oficiales.

\section{Nivel 1. Fase de reestructuración}

Destaca en esta fase la variedad de materiales y recursos didácticos preparados, especiaimente en la subfase de construcción de ideas (documentos históricos, noticias de prensa, bibliografía científico-didáctica, MAV, resolución de problemas, salidas...), con el fín de poder seleccionar los que sean necesarios según la dinámica concreta que surja en el aula y en función de variables clásicas como nứmero de alumnos, ideas previas sobre el módulo y tiempo disponible. La utilización de estos materiales, así como su forma de aplicación en el proceso, supone lógicamente la adquisición de contenidos en el ámbito didáctico.

Nivel 2. Fase de aplicación de ideas, ejemplos de materiales y recursos didácticos

- Análisis de programas oficiales y libros de texto de ES. En grupos de trabajo, se realizan una revisión de estos materiales en base a la pregunta: ¿Cómo es tratado el estudio de la «energía»?. Antalizan los resultados y contrastan con la secuenciación de contenidos y proceso seguido en los niveles 1 y 2 de nuestro módulo. Pretendemos comprobar hasta que punto han asimilado, por un lado, la problemática didáctica sobre la enseñanza de la «energía» y, por otro, nuestra aportación metodológica para contribuir a su resolución.

- Actividades prácticas: Entrevistar a niños y adultos sobre ejemplos de clases de «energía» y categorizar las respuestas según criterios del grupo. Comprobanos así su capaciơd de tratamiento de la información.

\section{Nivel 3. Fase de reestructuración, subfase de situaciones de conflicto}

Se diseñan experiencias o situaciones didácticas que provoquen conflicto, utilizando artículos sobre diagnóstico de ideas previas y vídeos documentales sobre aspectos relacionados con la «energía». Así, por ejemplo, si en su propuesta hay ausencia de exploracion y explicitación de iđeas, la actividad a realizar consiste en plantear conceptos (energía, calor...) que supongan dificultad de aprendizaje debido a las concepciones alternativas de los alumnos, estableciendo un debate sobre las causas de dicha dificultad.

\section{Contenidos profesionales}

Las estrategias didácticas diseñadas para la formación práctica de nuestros alumnos se corresponden con las fases de aplicación y revisión de ideas del niyel 3 del programa del módulo. Destacamos algunos aspectos

\section{Aplicación de ideas}

a) Partimos del resultado de los trabajos realizados en las fases anteriores, y se procede al diseño de unidades didácticas por parte de los grupos de alumnos.

b) Selección de materiales y planificación de la intervención didáctica.

c) Aplicación en el aula.

Revisión de ideas

a) Trabajo de contrastación y evaluación de la aplicación en el aula. Se realiza en varias sesiones mediante puestas en común de las propuestas que hayan podido ser desarrolladas en el centro de prácticas.

b) Concienciación sobre el cambio conceptual y metodológico experimentado; revisión de todo el proceso de aprendizaje seguido en el módulo a través de debates, puestas en común y aportaciones significativas del profesorado desde el marco teórico que fundamenta su propuesta metodológica objeto del presente trabajo. 


\section{REFERENCIAS BIBLIOGRÁFICAS}

AAVV, 1987. Approaches to Teaching Energy. CLISP in the classroom. (Centre for Studies in Science and Mathematics Education. University of Leeds).

BACHELERIE, C., 1982. Le concept de l'energie chez le preadolescent. Tesis doctoral. (École des Phychologues Praticiens: Paris).

DRIVER, R,, 1988. Un enfoque constructivista para el desartollo del currículo en Ciencias, Enseñanza de las Ciencias, $6, \mathrm{pp}$. 109-121.

DRIVER, R. y WARRINGTON, L., 1985. Students'use of the principle of energy conservation in problem situations, $P$ hysic Education, 20, pp. 171-175.

DUIT, R., 1983. Energy conception held by students and consequences for science teaching, en Misconceptions in Science and Mathematics, pp. 316-321. (Ithaca).

ELLSE, M., 1988. Transferring not transforming energy, School Science Review, 69 (248), pp. 427-437.

FEYMAN, R,, 1971. Fisica. Vol. I: Mecánica, radiación y calor. (Ed. Fondo educativo interamericano SA).

GARCÍA GÓMEZ, J. et al., 1989. Análisis experimental de los conceptos sobre Energía en alumnos del Ciclo Superior de $\mathrm{EGB}$, Didátrica de las Ciencias Experimentales y Sociales. 1, pp. 43-51.
HIERREZUELO, J., 1986. Revisión bibliográfica sobre la enseñanza de la Energía, Enseñanza de las ciencias, 4 (3), pp. 266-268.

KOBALLA,T., 1989. Using salient beliefs in designing a persuasive message about teaching energy conservation practices to children, Science Education, 73, pp. 547-567.

OSBORNE, R. y WITTROCK, M., 1985. The generative leaming model and its implications for Science Education,Studies in Science Education, 11, pp. 59-87.

SEVILLA, C., 1986. Reflexiones en torno al concepto de Energía. Implicacionescurriculares, Enseñanza de las Ciencias, 4 (3), pp. 247-252.

SEXL, R., 1981. Some observations concerning the Teaching of the Energy Concept, European Journal Science Education, 3, pp. 285-289.

SOLOMON, J., 1985. Teaching the conservation of energy, Physics Education, 20, pp. 165-170.

WATTS, D.M. y GILBERT, J.K., 1983. Enigmas in School Science students for scientifically associated words, Science and Technological Education, 1, pp. 161-171.

WARREN, J., 1982. The nature of energy, European Journal of Science Education, 4, pp. 295-297. 